\title{
REPORT OF THE TREASURER FOR THE PERIOD DECEMBER 1, 1936 TO NOVEMBER 30, 1937
}

\section{BALANCE SHEET}

November 30, 1937

Cash in Banks:

ASSETS

Corn Exchange Bank Trust Company ... \$ 3,579.88

Bank for Savings ................... 2,727.35

Union Dime Savings Bank ............. $5,868.30$

Investments (Value based on market quotation as of November $30,1937, \$ 90,396.75$.)

$\$ 12,175.53$

Total Assets

RESERVES :

RESERVES AND SURPLUS

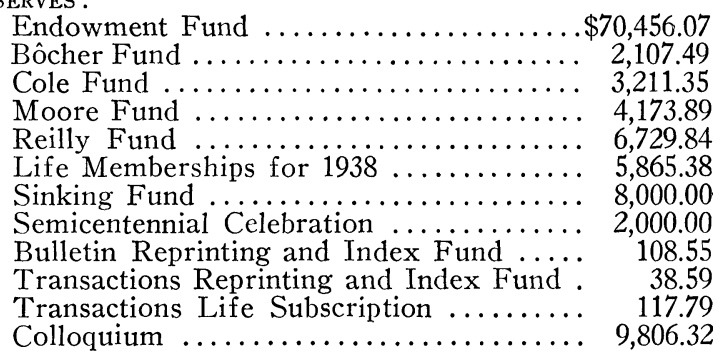

Surplus at November 30, 1937

Total Reserves and Surplus

STATEMENT OF GENERAL RECEIPTS AND GENERAL DISBURSEMENTS

GeNerAL Receipts :

Dues from Ordinary Memberships ............. $\$ 12,873.54$

Dues from Contributing Memberships ................. 1,201.97

Dues from Institutional Memberships ............. $6,429.75$

Initiation Fees .............................. ${ }^{6} 400.42$

Interest Income from Endowment Fund and General Investments $\ldots \ldots \ldots \ldots \ldots \ldots \ldots \ldots \ldots \ldots \ldots \ldots \ldots, 4,061.88$

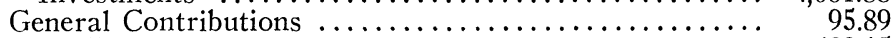

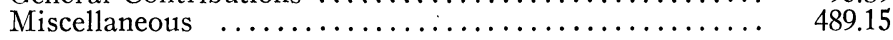

Total General Receipts

General Disbursements :

Secretaries

$\$ 5,200.00$

Treasurer

613.98

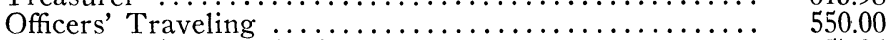

Office Furniture and Fixtures ................. 57.96

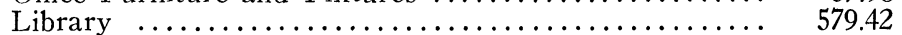

Membership Committee ...................... 200.00

Semicentennial Committee ......................... 100.00 
Income Collection Expense ..................... 265.24

American Journal Subvention $\ldots \ldots \ldots \ldots \ldots \ldots \ldots \ldots \ldots, 2,500.00$

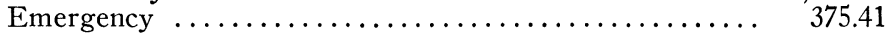

Total General Disbursements .............. 10,442.01

Excess of General Receipts over General Disbursements $\$ \$ \$ 15,110.59$

Surplus at December $1,1936 \ldots \ldots \ldots \ldots \ldots \ldots \ldots \ldots \ldots . \ldots \ldots \ldots$

Additions :

Adjustment for Amortization of Investments ........\$3,576.10

Adjustment of Prior Year's Income Distribution ...... 20.54

Adjustment of Life Membership Reserve ............ 250.53

Adjustment of Transactions Life Subscription Reserve $\quad .27$

Excess of General Receipts over General Disbursements

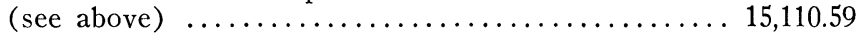

Total $\ldots \ldots \ldots \ldots \ldots \ldots \ldots \ldots \ldots \ldots \ldots \ldots \ldots$

Deductions :

Appropriation to Bulletin Account ..............\$ 7,981.16

Appropriation to Transactions Account ................ 2,546.27

Appropriation to Colloquium Account ............. 1,250.00

Appropriation to Semicentennial Fund ................ $1,000.00$

Appropriation to Sinking Fund $\ldots \ldots \ldots \ldots \ldots \ldots \ldots, 3,000.00$

Surplus at November $30,1937 \ldots \ldots \ldots \ldots \ldots \ldots \ldots \ldots$

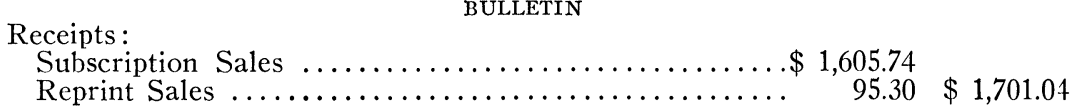

Appropriation from General Receipts ............. $7,981.16$

Total .................................. \$

Disbursements :

Printing (2 numbers for 1936, 10 for 1937) .........\$ 6,295.31

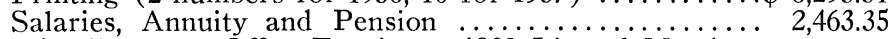

Miscellaneous-Office Furniture, 1938 List of Members

Expenses, Supplies, etc. .................... 839.54

Editors' Traveling ........................... 84.00

BULLETIN REPRINTING AND INDEX

Balance at December $1,1936 \ldots \ldots \ldots \ldots \ldots \ldots \ldots \ldots$

Receipts :

Back Volume Sales

339.85

Total .

$\$ 976.98$

Disbursements :

Reprinting

868.43

Balance at November 30, 1937

$\$ 108.55$

Receipts :

TRANSACTIONS

Subscription Sales ............................\$4,694.10

Back Volume Sales ........................ $\quad 785.75$

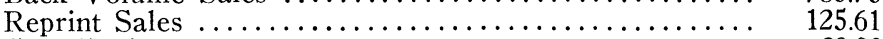

Contributions $\ldots \ldots \ldots \ldots \ldots \ldots \ldots \ldots \ldots \ldots \ldots \ldots, \quad 20.00$

Appropriation from General Receipts .............. 2,546.27

Total $\ldots \ldots \ldots \ldots \ldots \ldots \ldots \ldots \ldots \ldots \ldots \ldots \ldots . \quad \$ 8,171.73$ 
Disbursements :

Printing ( 1 number for 1936, 5 for 1937) .......\$6,169.45

Salaries, Annuity and Pension ............... 1,502.00

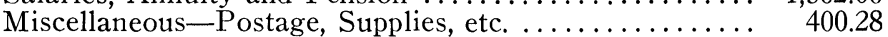

Editors' Traveling ........................ 100.00

TRANSACTIONS REPRINTING AND INDEX

Balance at December 1, 1936

Disbursements :

Reprinting

$\$ \quad 108.59$

70.00

$\$ \quad 38.59$

Balance at November 30, 1937

\section{Colloguium}

Balance at December $1,1936 \ldots \ldots \ldots \ldots \ldots \ldots \ldots \ldots \ldots \ldots$

Receipts :

Sales of Colloquium Volumes .............\$2,267.45

Sales of Colloquium Vo'ume 16 (Bliss) for National

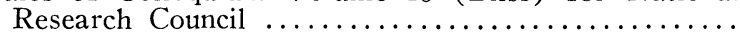

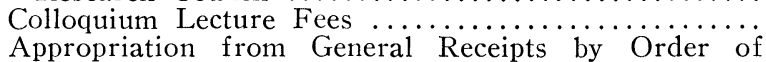

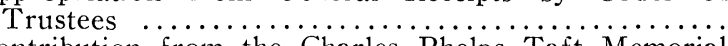

Contribution from the Charles Phelps Taft Memorial

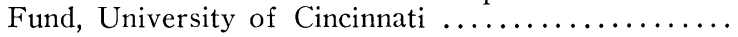

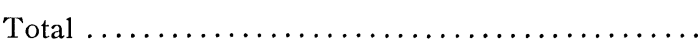

Disbursements:

$1,250.00$

$+\frac{600.00}{\$ 12,379.33}$

Printing (Volume 21) .................\$ 1,038.52

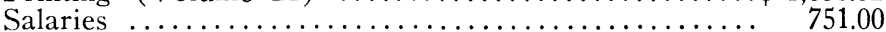

Miscellaneous-Shipping, Supplies, etc. (including cost of shipping Volume 16 for National Research Council)

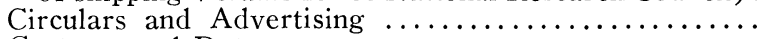

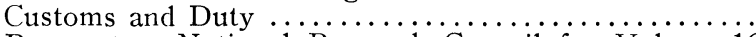

Payment to National Research Council for Volume 16

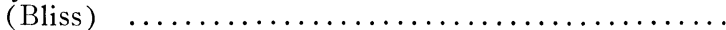

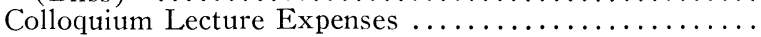

305.78

68.06

270.69

89.20

49.76

$2,573.01$

$\$ 9,806.32$

Balance at November 30, 1937

Endow ment Fund

SPECIAL FUNDS

Balance at December 1, 1936

Total Principal

Income Additions :

Income and Gifts ............ 2,538.76 $\$ 70,431.07 \$ 70,431.07$

Adjustment for Amortization of Investments 3,174.27

Total

$\$ 76,144.10$

$25.00 \$ 2,513.76$ $3,174.27$

$\overline{\$ 70,456.07} \overline{\$ 5,688.03}$ Deduction:

Transferred to General Receipts and Surplus 5,688.03

$5,688.03$

Balance at November 30, 1937 $\$ 70,456.07 \quad \$ 70,456.07$

Bôcher Fund

Balance at December 1, 1936 $. \$ 1,955.49 \$ 1,188.00$

$\$ 767.49$

Additions :

Income

Adjustm

69.35

69.35

87.33

87.33

Total 
Deduction :

Adjustment of Prior Year's Income Distri-

bution ............................

Balance at November $30,1937 \ldots \ldots \ldots \ldots \ldots . \overline{\$ 2,107.49} \overline{\$ 1,188.00} \overline{\$ 919.49}$

Cole Fund

Balance at December 1, 1936

$\$ 2,093.13 \quad \$ 984.92$

Additions :

Income .......................... 108.35

108.35

Adjustment for Amortization of Invest-

ments

32.23

Total

$\$ 3,218.63$

32.23

eduction:

Adjustment of Prior Year's Income Distri-

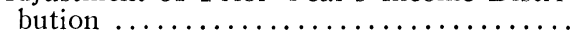

7.28

$\overline{\$ 2,093.13} \overline{\$ 1,125.50}$

$\begin{array}{r}7.28 \\ \$ 3,211.35 \\ \hline\end{array}$

Balance at November 30, 1937

\$ $4,030.39 \quad \$ 2,100.62 \quad \$ 1,929.77$

Moore Fund

Balance at December 1, 1936

Additions :

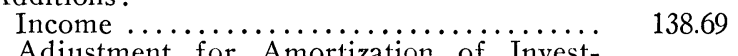

138.69

Adustment for Amortization of Invest-

13.39

13.39

Total

$\overline{\$ 4,182.47} \overline{\$ 2,100.62} \overline{\$ 2,081.85}$

Deduction :

Adjustment of Prior Year's Income Distri-

bution

8.58

8.58

Balance at November 30, 1937

$\overline{\$ 4,173.89} \overline{\$ 2,100.62} \overline{\$ 2,073.27}$

ReIlly Fund

Balance at December $1,1936 \ldots \ldots \ldots \ldots \ldots . \$ 6,558.26$ \$ $6,531.00$ \$ 27.26

Addition :

Income $\ldots \ldots \ldots \ldots \ldots \ldots \ldots \ldots \ldots \ldots \ldots+221.04 \quad 221.04$

Total $\ldots \ldots \ldots \ldots \ldots \ldots \ldots \ldots \ldots \ldots . \overline{\$ 6,779.30} \overline{\$ 6,531.00} \overline{\$ 248.30}$

Deduction:

Adjustment for Amortization of Invest-

ments

49.46

49.46

Balance at November 30, 1937

$\overline{\$ 6,729.84} \overline{\$ 6,531.00} \overline{\$ 198.84}$

P. A. Smith

New York, N.Y.

Treasurer

November 30, 1937 